\title{
Editorial
}

\section{Fundamental Issues in Mobile Healthcare Information Systems}

\author{
Basit Shahzad, ${ }^{1}$ Mehmet A. Orgun, ${ }^{2,3}$ and Christoph Thuemmler ${ }^{4}$ \\ ${ }^{1}$ College of Computer \& Information Science, King Saud University, Riyadh 12372, Saudi Arabia \\ ${ }^{2}$ Intelligent Systems Group (ISG), Department of Computing, Macquarie University, Sydney, NSW 2109, Australia \\ ${ }^{3}$ Faculty of Information Technology, Macau University of Science and Technology, Avenida Wai Long, Taipa 999078, Macau \\ ${ }^{4}$ Edinburgh Napier University, Edinburgh EH10 5DT, UK
}

Correspondence should be addressed to Mehmet A. Orgun; mehmet.orgun@mq.edu.au

Received 25 July 2016; Accepted 25 July 2016

Copyright (C) 2016 Basit Shahzad et al. This is an open access article distributed under the Creative Commons Attribution License, which permits unrestricted use, distribution, and reproduction in any medium, provided the original work is properly cited.

\section{Introduction}

Consistent growth in the mobile technology has led to many significant features that have improved the quality of services provided to people from all walks of life. While the need for a broader and more diverse range of available services is growing, the multifaceted and deep rooted challenges of the mobile technology such as security, privacy, efficiency, coherence, and resilience have to evolve. While most of the online businesses, social networking, financial transactions, personal record managements, and so forth are increasingly being done from mobile phones, it is important that an appropriate infrastructure capable of handling this data is in place to keep, handle, update, secure, and make it available when needed in accordance with national and international rules and regulations. The quality of life can be improved by automating a number of tasks that have a direct impact on day-to-day living. One prominent area of growing interest in this regard is the support of healthcare provision anywhere, anyhow, and at any time. Suitable information systems and the relevant network infrastructures are moving closer and closer together. There is the need to have security, privacy, efficiency, robustness, consistency, and availability at all times. In order to incorporate the said objectives, it is justified to advocate an information system that can operate on mobile devices to provide healthcare services, whereby the service layer and the data transportation layer are moving increasingly closer to each other.

Healthcare systems are at the cusp of being revolutionized by advancements in technology, which when appropriately integrated into existing best practices can enable faster and safer cure, improved doctor-patient relationships, personalized treatments, and lower costs. Typically this can be measured and controlled by monitoring the Quality of Experience (QoE).

With rapid advances in computing and associated technologies, we are also seeing steady and seamless integration of communication, networking, hardware miniaturization, sensing, cryptography, and a range of algorithmic advances for smarter and increasingly personalized healthcare. At the forefront of challenges in emergent smarter healthcare systems the issues are how to manage massively growing amount of healthcare information and smart devices over a variety of technologies across different domains maintaining a guaranteed quality of service (QoS) at any time.

In the realm of healthcare, while there are clear opportunities to leverage information management emanating from today's computing technologies, additional challenges include providing information reliability, security, patient's privacy, real-time criticality, information fusion, system sustainability, and social interaction, among others. Although research in the domain of information management analytics for smarter healthcare is attracting attention across disciplines, critical applications, new opportunities, challenges, models, and technologies are yet to be explored and investigated.

\section{Contributions}

The special issue attracted 19 contributions in total. After an extensive review period involving the distinguished expert 
reviewers and the guest editors, five papers were accepted for the special issue, resulting in an acceptance rate of $26 \%$. The introduction of the accepted papers is presented below.

The first paper, titled "Analysis of Denial of Service Impact on Data Routing in Mobile eHealth Wireless Mesh Network," by S. Alanazi et al., states that wireless mesh networks (WMNs) are a promising technology that has emerged with the combination of several wireless networks. These wireless networks and devices communicate in a mesh network manner, to provide edge-to- edge, easy, and cost-effective data communication. Many current and future promising applications depend on WMNs and one of the most important is eHealth, where the confidential information is transmitted with the help of WMNs. The authors state that denial of service (DoS) attacks are fatal to many types of networks, including wireless mesh networks, specifically when the network is utilized in a highly sensitive scenario like eHealthcare. This paper analyzes three types of attacks that can cause DoS in static and mobile WMNs and the remedies against them.

The second paper, titled "A Case of Engineering Quality for Mobile Healthcare Applications Using Augmented Personal Software Process Improvement," by S. A. K. Ghayyur et al., starts with the discussion that mobile healthcare systems are currently considered among the key research areas in the domain of software engineering. Modern technologies, for mobile healthcare systems, are readily available. The authors present the Architecture Augmented Personal Process technique in order to enhance the quality of mobile healthcare systems through the use of an architectural design with an integration of the personal software process.

The third paper, titled "Integrated Wearable System for Monitoring Heart Rate and Step during Physical Activity," by E. A. P. J. Prawiro et al., proposes integrating a heart rate (HR) monitoring system with a step counter for use during physical activities. A novel step counter algorithm has been developed to enable the highly accurate detection of a step. The proposed system comprises a wireless wearable device, a smartphone, and a remote server. Data transmission between a wearable device and a smartphone is conducted via Bluetooth low energy (BLE). An indirect contact measurement method has also been devised to eliminate the need for direct contact electrodes and the likelihood of skin irritation. The proposed system is compact, lightweight, and comfortable to wear. A smartphone application provides the interface for the display of data related to $\mathrm{HR}$, step count (SC), exercise intensity, speed, distance, and calories burned, as well as waveforms related to ECG and step cycle. The ECG peak detection algorithm achieved an accuracy of $99.7 \%$ using the MIT-BIH ST change database. An accuracy of $98.89 \%$ was achieved for HR and $98.96 \%$ for SC at treadmill speeds of 1.8 to $9.0 \mathrm{~km} / \mathrm{h}$.

The fourth paper, titled "Automatic Gender Detection based on Characteristics of Vocal Folds for Mobile Healthcare System," by M. Alhussein et al., proposes that automatic gender detection may be useful in some applications of a mobile healthcare system. In a human voice production system, the contribution of the vocal folds is very vital. The length of the vocal folds is gender dependent; a male speaker has longer vocal folds than a female speaker. Due to longer vocal folds, the voice of a male speaker becomes heavy and, therefore, contains more voice intensity. Based on this idea, a new type of a time domain acoustic feature for an automatic gender detection system is proposed in this paper. The proposed feature measures the voice intensity by calculating the area under the modified voice contour to make the differentiation between males and females. Two different databases are used to show that the proposed feature is independent of text, spoken language, dialect region, recording system, and environment. The experimental results show that the detection rates for clean and noisy speech are $98.27 \%$ and $96.55 \%$, respectively.

The fifth and the last paper, titled "EVFDT: An Enhanced Very Fast Decision Tree Algorithm for Detecting Distributed Denial of Service Attack in Cloud-Assisted Wireless Body Area Network," by R. Latif et al., states that the detection denial of service attacks demands an adaptive and incremental learning classifier capable of accurate decision making with less computation. The DDoS attack detection using existing machine learning techniques requires the full data set to be stored in the memory and is not appropriate for real-time network traffic. To overcome these shortcomings, the Very Fast Decision Tree (VFDT) algorithm has been proposed in the past that can handle high speed streaming data efficiently. While considering the data generated by WBAN sensors, noise is an obvious aspect that severely affects the accuracy and increases false alarms. In this paper, an enhanced VFDT (EVFDT) algorithm is proposed to efficiently detect the occurrence of DDoS attacks in cloudassisted WBANs. EVFDT uses an adaptive tie-breaking threshold for node splitting. To resolve the tree size expansion under extreme noise, a lightweight iterative pruning technique is proposed. To analyze the performance of EVFDT, four metrics are used: classification accuracy, tree size, time, and memory. Simulation results show that EVFDT attains a significantly high detection accuracy with fewer false alarms.

\section{Acknowledgments}

We are grateful to the authors who submitted their valuable contributions for consideration in this special issue, and the reviewers who generously donated their time in the review process. Without their help and contributions, this special issue would not have been possible. We hope that the readers will find the papers in this special issue stimulating.

Basit Shahzad Mehmet A. Orgun Christoph Thuemmler 

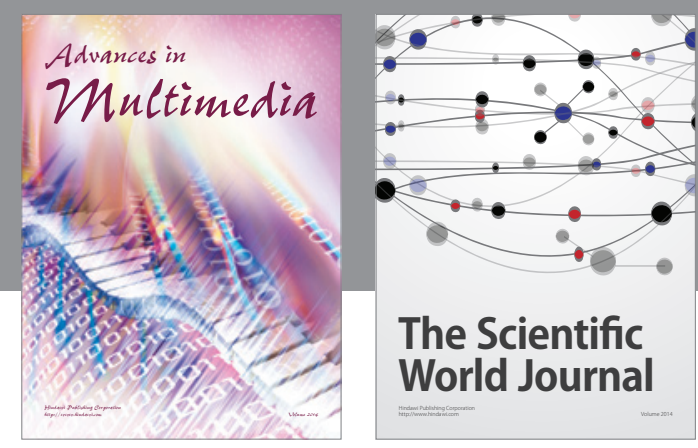

The Scientific World Journal
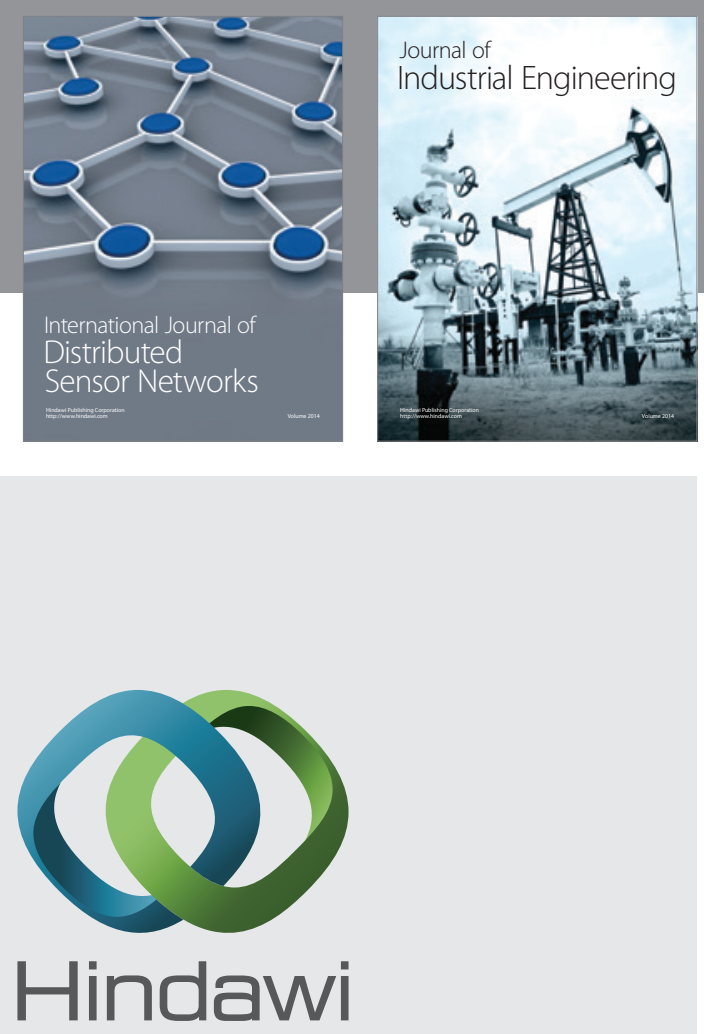

Submit your manuscripts at

http://www.hindawi.com

\section{Computer Networks} and Communications
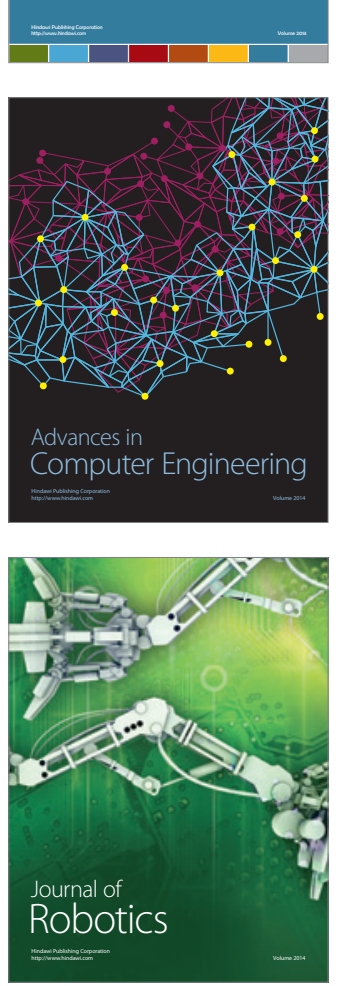
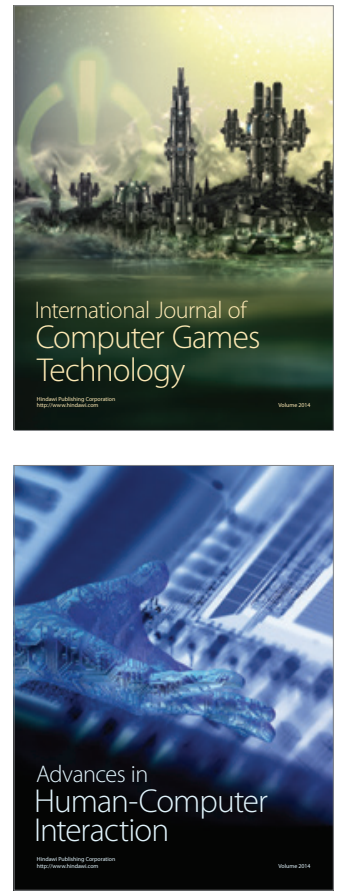
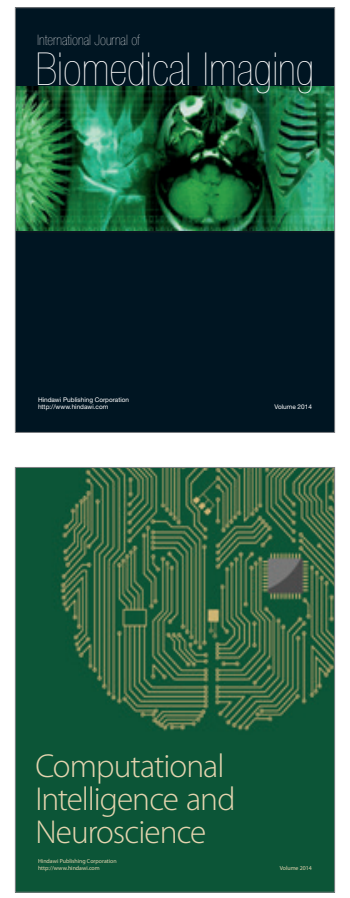
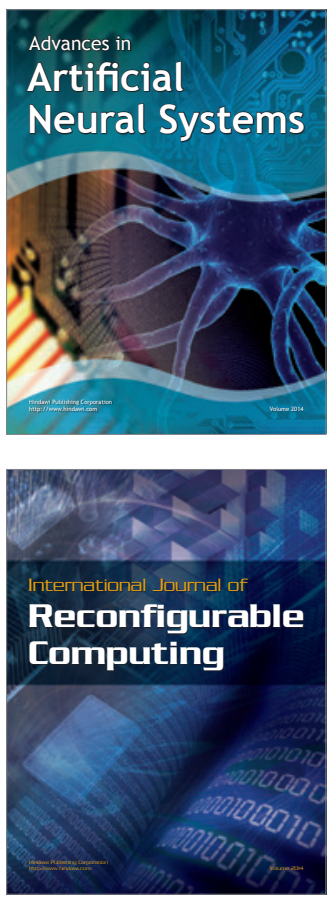
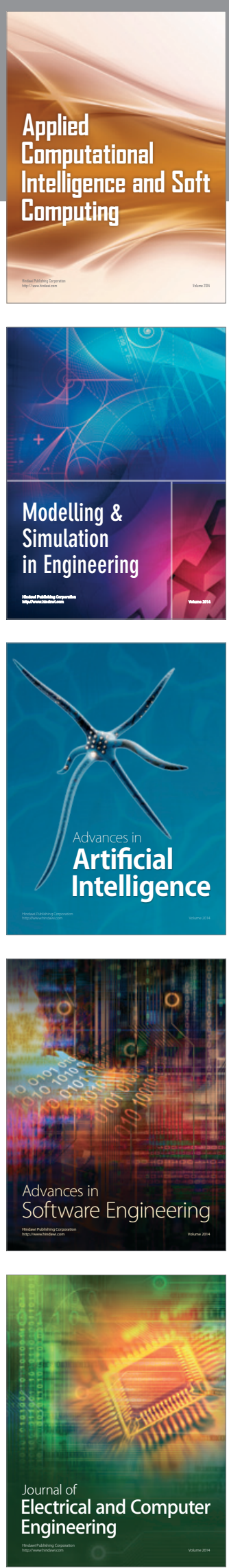